\title{
Epidemiologia da raiva em herbívoros domésticos em uma localidade na Amazônia brasileira
}

A raiva é uma das zoonoses com grande impacto tanto no desenvolvimento do agronegócio, quanto na Saúde Pública. Casos são registrados com frequência na Amazônia brasileira, Norte do Brasil, como na mesorregião Nordeste do Estado do Pará, considerada área focal para estudos relacionados à raiva transmitida por morcegos hematófagos. Objetivo: avaliar a distribuição temporal da raiva em herbívoros domésticos por um período de 13 anos no município de Bragança/PA e verificar a relação entre os fatores pecuária e desmatamento. Metodologia: os dados foram obtidos de fontes governamentais oficiais: casos de raiva em herbívoros (ADEPARÁ), produção pecuária (IBGE) e desmatamento (INPE/PRODES) e para análise destes dados utilizou-se os programas Microsoft Excel@ 2013 e BioEstat 5.3. Resultados: as notificações da doença tiveram maior percentual em bovídeos, com maior concentração de casos na estação mais chuvosa, foi observado tendência de crescimento de amostras para diagnóstico laboratorial, bem como de casos positivos de raiva no período de estudo. Além de forte correlação entre pecuária e desmatamento. Conclusões: a intensa antropização da área chega até $81 \%$ em desmatamento e o aumento do rebanho bovídeo, revelaram forte correlação desta questão ambiental. Sugere-se ciclicidade e sazonalidade da doença. Possivelmente a produção pecuária e o desmatamento, além de outros, isolados ou cumulativamente, a exemplo da bio-ecologia dos morcegos, possam estar contribuindo para a ocorrência da doença nesta localidade da Amazônia brasileira.

Palavras-chave: Epidemiologia; Raiva; Quirópteros; Desmatamento; Amazônia.

\section{Epidemiology of rabies in domestic herbivores in a location in the Brazilian Amazon}

\begin{abstract}
Rabies is one of the zoonoses with great impact both on agribusiness development and on Public Health. Rabies cases are frequently witnessed in the Brazilian Amazon, Northern Brazil, as well as in the Northeast mesoregion of Pará, which is considered a focal area for studies related to rabies transmitted by hematophagous bats. Objective: evaluate the temporal distribution of rabies in domestic herbivores for a period of 13 years in Bragança/PA and verify the relationship between livestock and deforestation factors. Methodology: data were obtained from official government sources: cases of rabies in herbivores (ADEPARÁ), livestock production (IBGE) and deforestation (INPE/PRODES). In order to analyse data, Microsoft Excel@ 2013 and BioEstat 5.3 were used. Results: disease notifications had a higher percentage in bovines, with a higher concentration of cases in the rainiest season. A growth trend of samples for laboratory diagnosis was observed, as well as positive cases of rabies in the investigation period. In addition to the strong correlation between livestock and deforestation. Conclusions: The intense anthropization of the area reaches up to $81 \%$ in deforestation and the increase in the cattle herd, has revealed a strong correlation of this environmental issue. Cyclicality and seasonality of the disease are suggested. Possibly livestock production and deforestation, in addition to others, isolated or cumulatively, like bats' bio-ecology, may be contributing to the occurrence of the disease in this location in the Brazilian Amazon.
\end{abstract}

Keywords: Epidemiology; Rabies; Chiropterans; Deforestation; Amazon.

Topic: Epidemiologia e Saúde Ambiental

Reviewed anonymously in the process of blind peer.
Received: 17/03/2020

Approved: 18/04/2020
Lucila Pereira da Silva (iD)

Universidade do Estado do Pará, Brasil

http://lattes.cnpq.br/9588660640610043

http://orcid.org/0000-0003-0040-9482

lucilapsilva@yahoo.com.br

Ana Paula Vilhena Beckman Pinto (iD

Universidade do Estado do Pará, Brasil

http://lattes.cnpq.br/6058277301641587

http://orcid.org/0000-0001-5673-7691

anabeckman@yahoo.com.br

Altem Nascimento Pontes (iD)

Universidade do Estado do Pará, Brasil

http://lattes.cnpq.br/5993352890364998

http://orcid.org/0000-0002-9001-4603

altempontes@hotmail.com

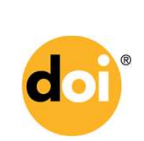

DOI: 10.6008/CBPC2179-6858.2020.003.0010

\author{
Cléa Nazaré Carneiro Bichara (in \\ Universidade do Estado do Pará, Brasil \\ http://lattes.cnpq.br/2161704040280760 \\ http://orcid.org/0000-0002-2995-0136 \\ cleabichara@ig.com.br
}

Referencing this:

SILVA, L. P.; PINTO, A. P. V. B.; PONTES, A. N.; BICHARA, C. N. C.. Epidemiologia da raiva em herbívoros domésticos em uma localidade na Amazônia brasileira. Revista Ibero Americana de Ciências Ambientais, v.11, n.3, p.105-112, 2020. DOI:

http://doi.org/10.6008/CBPC2179-6858.2020.003.0010 


\section{INTRODUÇÃO}

A raiva é considerada uma das mais graves zoonoses do mundo, cujo agente etiológico é um vírus do gênero Lyssavírus, família Rhabdoviridae. Tem grande impacto na Saúde Pública, sobretudo pela sua evolução letal, como também pelo elevado custo social e econômico (TEIXEIRA et al., 2015). Os prejuízos econômicos causados por esta enfermidade, principalmente a raiva dos herbívoros, são em torno de 44 milhões por ano; a mortalidade é de cerca de 50.000 cabeças de gado, além das perdas indiretas (SILVA et al., 2017).

As alterações ambientais, como o desmatamento afetam negativamente os ecossistemas naturais muitas dos quais interferem no bem-estar e saúde do próprio homem, bem como na dinâmica de muitas doenças, entre as quais a raiva, cujo agente transmissor, seus hospedeiros, incluindo o homem têm forte ligação com alteração ambiental (ALHO, 2012). O morcego hematófago Desmodus rotundus é o principal transmissor da doença aos herbívoros domésticos, pois constituem sua fonte alimentar mais frequente (ANDRADE et al., 2016).

No ambiente rural os herbívoros domésticos são hospedeiros acidentais do vírus da raiva, pois, apesar de participarem da cadeia epidemiológica da raiva rural, somente contribuem como sentinelas à circulação do vírus (SOUZA et al., 2014). Nestes animais, a raiva é considerada endêmica em diversas regiões do Brasil (PUGA, 2015), a exemplo da região Norte (RIBEIRO et el., 2018), possivelmente relacionada à ação antrópica caracterizada pelo desmatamento em densas florestas, o que se considera um dos principais fatores que contribuem para a mudança da dinâmica da raiva por proporcionarem a dispersão dos morcegos dos abrigos naturais e a disseminação do vírus (BRAGA et al., 2014). Sabe-se que existe uma importante relação entre o número de casos de raiva e o aumento da produção bovina nesta mesorregião, devido o gado ser uma fonte farta de alimentação e uma presa de fácil acesso para os quirópteros (FERNANDES et al., 2013).

No Brasil há programas governamentais responsáveis pelas ações de controle da raiva em herbívoros, tanto à nível federal, quanto estadual (BRASIL, 2009) e que no Pará é executado pela Agência de Defesa Agropecuária do Estado do Pará - ADEPARÁ, tais ações de controle incluem vigilância epidemiológica, vacinação dos herbívoros domésticos, controle populacional do morcego hematófago $D$. rotundus e educação em saúde (PARÁ, 2004).

Nos últimos anos, a mesorregião Nordeste Paraense tornou-se uma área focal para estudos de quirópteros e da raiva nos mais diversos aspectos relacionados a doença, pois tem sido considerada a região da principal circulação do vírus da raiva (COSTA, 2011), o que justifica a escolha territorial para a abordagem proposta, quanto a avaliação da distribuição temporal da raiva em herbívoros, visando subsidiar as ações de controle da raiva nas espécies de interesse econômico, além de contribuir com o aumento do conhecimento sobre a epidemiologia da raiva na Amazônia brasileira.

\section{METODOLOGIA}

Fazendo parte da Amazônia brasileira o município de Bragança (Figura 1) pertence a mesorregião 
Nordeste Paraense, localizada na microrregião Bragantina (01 $01^{\prime} 15^{\prime \prime}$ S e $46^{\circ} 46^{\prime} 10^{\prime \prime}$ W) com população estimada de 126.436 pessoas (IBGE, 2018a) em uma área de $2.091,90 \mathrm{~km}^{2}$, constituída em 18,84\% por floresta primária, 40,56\% de floresta secundária, $23,74 \%$ de pastagem e 0,60\% de área destinada a agricultura (CORDEIRO et al., 2017). O solo tem considerável trecho do tipo várzea, sendo o principal destaque da hidrografia o rio Caeté (FAPESPA, 2016). A classificação entre estação mais chuvosa (janeiro a agosto) e menos chuvosa (setembro a dezembro) obedecerá ao proposto por Moraes et al. (2005).

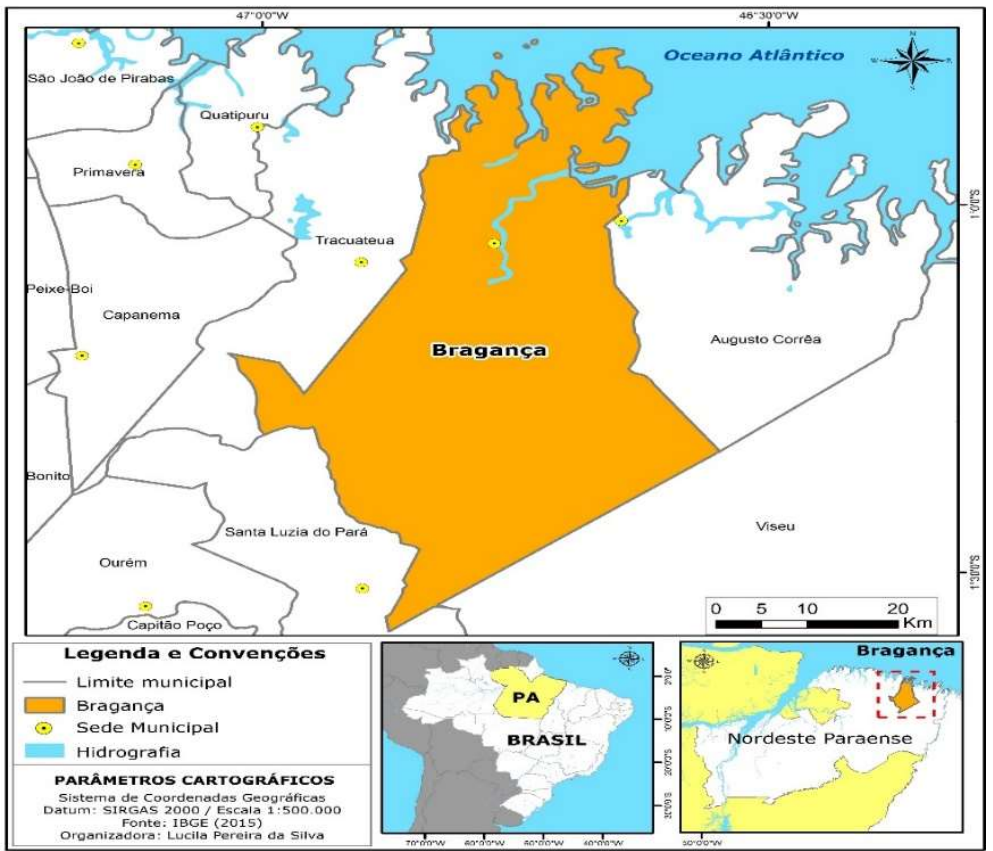

Figura 1: Localização geográfica do município de Bragança no Estado do Pará.

Os dados compreenderam de janeiro de 2006 a dezembro de 2018 e foram obtidos de fontes oficiais governamentais: casos de raiva (ADEPARÁ), produção pecuária (IBGE, 2018b) e desmatamento municipal anual (INPE, 2018). Foram realizadas as seguintes análises: tendência linear de amostras enviadas ao laboratório e de amostras positivas através do programa Microsoft Excel@ 2013; estatística descritiva da distribuição mensal dos casos positivos; correlação de Pearson entre desmatamento total e efetivo bovídeo através do programa BioEstat 5.3 (AYRES et al., 2007).

\section{RESULTADOS}

Foram analisadas laboratorialmente 26 amostras de Sistema Nervoso Central (SNC) de herbívoros suspeitos de raiva. A taxa geral de positividade para raiva foi de $57,7 \%(n=15 / 26)$, com maior ocorrência entre bovídeos, a saber bovinos e bubalinos (73,33\%) (Tabela 1). Não houve notificações de casos de raiva nas espécies suínas, caprinas e ovinas no período estudado.

Tabela 1: Distribuição de amostras de herbívoros suspeitos de raiva de acordo com as espécies e positividade no município de Bragança/PA no período de 2006 a 2018.

\begin{tabular}{|l|c|c|c|c|}
\hline \multicolumn{1}{|c|}{ Espécies afetadas } & $\begin{array}{c}\text { Amostras enviadas } \\
\text { para diagnóstico }\end{array}$ & Amostras positivas & $\begin{array}{c}\text { Percentual de } \\
\text { positividade }\end{array}$ & $\begin{array}{c}\text { Taxa de positividade } \\
\text { (\%) }\end{array}$ \\
\hline Bovídea & 17 & 11 & $73,33 \%$ & 64,7 \\
\hline Equídea & 9 & 4 & $26,67 \%$ & 44,44 \\
\hline Total & 26 & 15 & $100,00 \%$ & 57,7 \\
\hline
\end{tabular}


Observou-se tendência de aumento do número de amostras enviadas para diagnóstico (Figura 2), assim como tendência de aumento de resultados positivos (Figura 3).

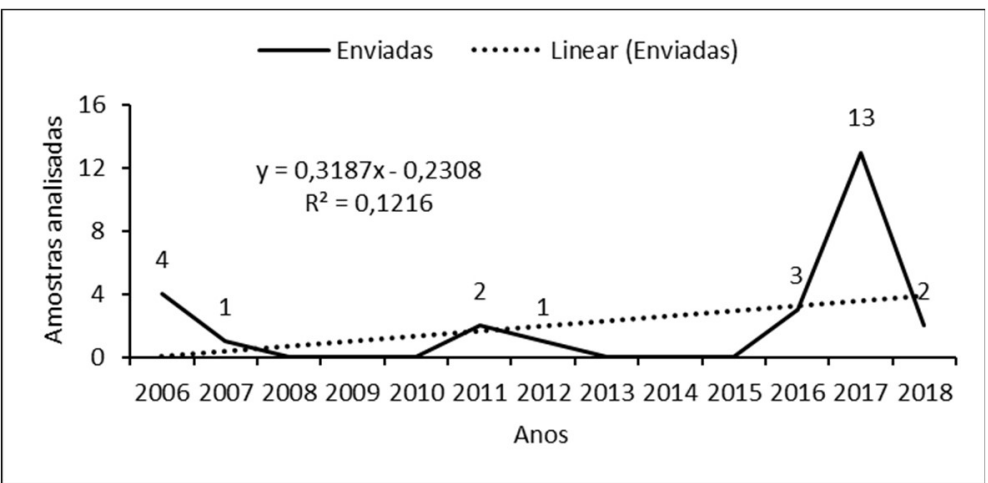

Figura 2: Tendência de envio de amostras para diagnóstico de raiva em herbívoros no município de Bragança/PA, no período de 2006 a 2018.

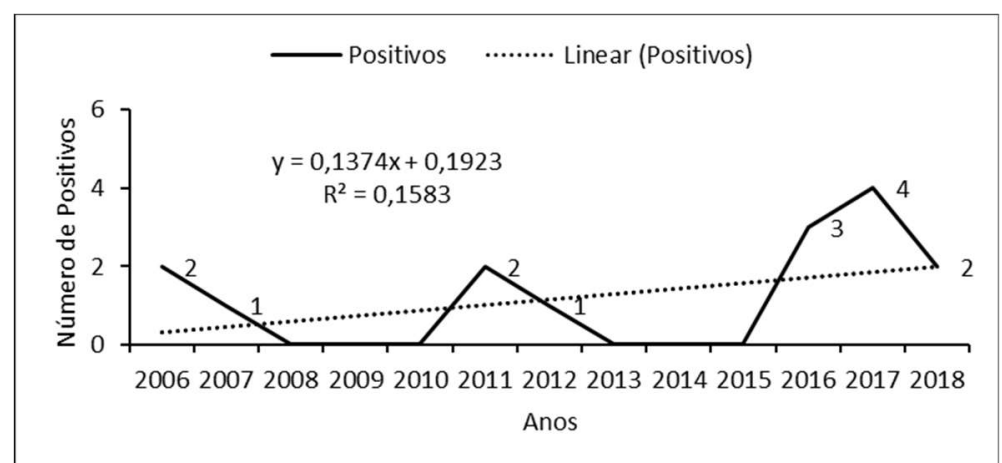

Figura 3: Tendência de diagnósticos positivos para raiva em herbívoros no município de Bragança/PA, no período de 2006 a 2018.

A distribuição mensal dos casos positivos ao longo da série (média=1,25; desvio padrão= 0,9653; variância $=0,9318)$ demonstrou que o maior número de casos de raiva em herbívoros $(n=11)$ ocorreu no período mais chuvoso (janeiro a agosto), com pico mais alto no mês de março. Notadamente, não houve registro de positividade nos meses de fevereiro, julho e setembro (Figura 4).

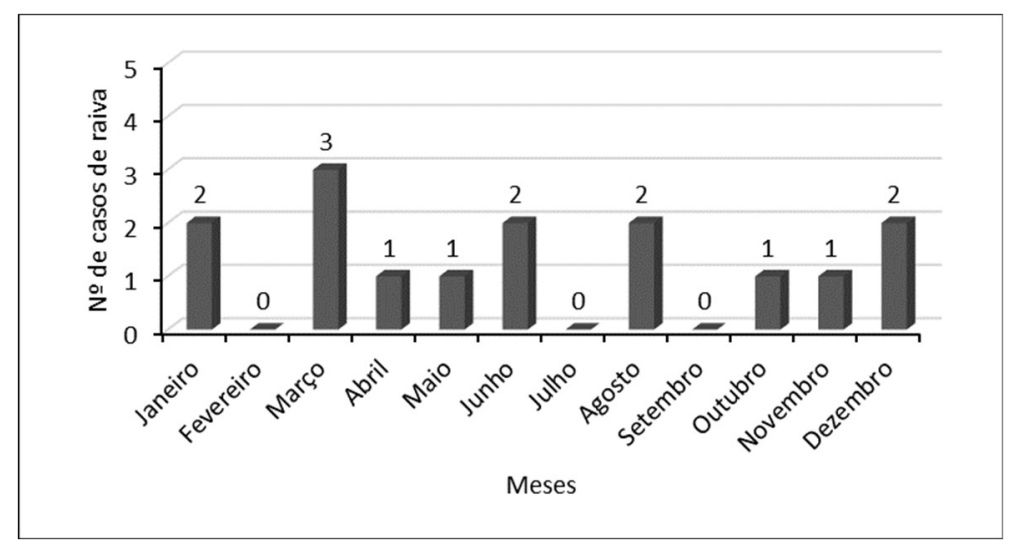

Figura 4: Distribuição mensal dos casos de raiva em herbívoros no município de Bragança/PA, no período de 20062018.

No período analisado houve expressivo crescimento da pecuária bovídea, passando de 21.955 cabeças (2006) para 30.025 cabeças (2018) e aumento do desmatamento, saindo de $1.711 \mathrm{~km}^{2}$ (2006) para $1.724 \mathrm{~km}^{2}$ (2018) (Figura 5). Observou-se forte correlação $(r=0,7418 ; p=0,0037)$ entre a pecuária bovídea e o 
desmatamento. Destaca-se que o desmatamento acumulado compreende cerca de $81 \%$ da área total do município (INPE, 2018).

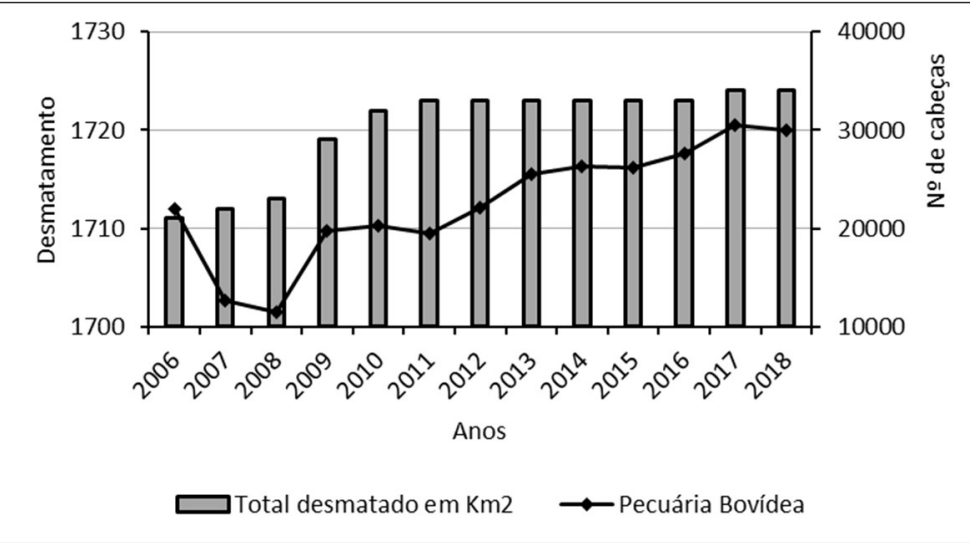

Figura 5: Evolução da pecuária bovídea e do desmatamento no município de Bragança/PA no período de 2006 a 2018. Fonte: IBGE (2018b); INPE (2018).

\section{DISCUSSÃO}

A maior ocorrência da raiva foi em bovídeos. Resultados compatíveis foram observados em um estudo na região Norte, na qual a maior taxa de prevalência (32,25\%) foi em bovídeos (CASSEB et al., 2006), assim como em estudos nacionais, a exemplo de Mato Grosso que apresentou taxa de prevalência em bovinos de 80\% (SILVA et al., 2017) e no Paraná de 86,91\% (DOGNANI et al., 2016). Isto provavelmente foi devido ao fato de a população bovina estar em maior número que as demais espécies de animais de produção (RIBEIRO et al., 2018).

Analisando conjuntamente o aumento de amostras enviadas para diagnóstico e o aumento de resultados positivos, poderia indicar possíveis falhas no controle da doença por parte da defesa sanitária animal no estado. Porém, a elevação de casos positivos acompanhada logo em seguida de períodos redução ou ausência da doença, conforme apresentado na figura 3, parece indicar que as ações de controle da doença foram eficazes a ponto de ocorrerem 2 períodos de 3 anos consecutivos sem notificação da doença. Cabe salientar que nenhum método eliminou a circulação do vírus da raiva, mesmo naquelas áreas onde o controle de morcegos hematófagos é realizado regularmente (BLACKWOOD et al., 2013).

Os resultados deste trabalho foram compatíveis com o que é observado na América do Sul, onde a raiva dos herbívoros ocorre em surtos cíclicos, reaparecendo com periodicidade de 3-7 anos, obedecendo também ao ciclo da raiva nas colônias de morcegos quando ocorre um pico da doença, um período de declínio, para repovoamento e reinfecção das colônias de morcegos (MARCOLONGO-PEREIRA et al., 2011). Porém, outros autores não concluíram pela existência de ciclicidade na região Norte, embora tenha ocorrido aumento de casos de raiva a cada 2, 3 ou 5 anos em alguns estados (RIBEIRO et al., 2018).

Considerando que o maior número de casos de raiva ocorreu no período mais chuvoso julgou-se importante analisar a possibilidade de sazonalidade da doença. Um padrão semelhante ao observado no presente estudo foi observado na raiva humana e animal no estado do Pará durante 7 anos, onde o pico mais alto da doença foi no mês de março, considerado mais chuvoso (LIMA, 2006). Assim também outra pesquisa 
realizada em Bragança/PA encontrou maior percentual de soropositividade em morcegos na estação chuvosa. Estes exemplos são importantes, pois representam compatibilidade com os resultados encontrados neste trabalho, sugerindo sazonalidade da raiva na região estudada. Mas outros estudos nacionais no Paraná e São Paulo não encontraram padrão de sazonalidade para raiva (GOMES et al., 2011; SILVA et al., 2011).

Analisando a relação entre a pecuária bovídea e o desmatamento no município estudado, a forte correlação existente entre estas variáveis corrobora que a pecuária bovina é a atividade mais fortemente correlacionada com o desmatamento para os municípios da Amazônia (NEVES et al., 2014). O desmatamento anual em Bragança apresentou baixo incremento percentual, porém o acumulado chega a $81 \%$ em desmatamento (INPE, 2018), sendo preocupante considerar que áreas extensamente desmatadas, bem como naquelas com grandes rebanhos de gado e com maior número de rodovias são consideradas de maior risco para a transmissão da doença por morcegos hematófagos (ANDRADE et al., 2016).

Sabe-se que os casos de raiva estão associados ao aumento da produção bovina na mesorregião Nordeste Paraense, devido ao gado ser considerado uma fonte farta de alimentação para o morcego e uma presa de fácil acesso, existindo uma importante relação entre o número de casos de raiva e o aumento da produção bovina (FERNANDES et al., 2013). Nesse contexto, Mayen (2003) associa a emergência do ciclo silvestre da raiva com áreas de ocorrência de impactos ambientais negativos, pois o aumento da produção bovina está diretamente relacionado com a conversão da cobertura vegetal em pasto. Porém, Diogo Filho (2018) em Goiás, não conseguiu explicar o comportamento da raiva bovina, considerando o uso da terra e efetivo bovino, indicando que possivelmente outros fatores, como a presença de morcegos hematófagos, abrigos artificiais e os distúrbios ambientais possam ter influenciado mais diretamente na distribuição da doença.

\section{CONCLUSÕES}

A ocorrência da raiva em herbívoros domésticos revela que o vírus da raiva circula nesta localidade, reforçando a importância de que eventos-sentinela sejam continuamente monitorados tendo em vista o risco da ocorrência da raiva em humanos, fatos já vivenciados na Amazônia brasileira. Sugere-se a existência de sazonalidade e de ciclicidade na área estudada, mas necessitando de estudos mais aprofundados para sua confirmação.

A forte correlação encontrada entre pecuária bovídea e desmatamento não deve ser descartada, pois tanto o incremento do rebanho bovino quanto o desmatamento da floresta podem contribuir para a ocorrência da raiva em bovinos. Possivelmente, além da questão ambiental, outros fatores, a exemplo da bio-ecologia dos morcegos, possam estar contribuindo efetivamente para a ocorrência da doença nesta localidade.

AGRADECIMENTOS: ao Programa de Pós-graduação em Ciências Ambientais da Universidade do Estado do Pará. 


\section{REFERÊNCIAS}

ALHO, C. J. R.. A importância da biodiversidade para a saúde humana: uma perspectiva ecológica. Estudos Avançados, v.26, n.74, p.151- 165, 2012. DOI: https://doi.org/10.1590/S0103-40142012000100011

AYRES, M.; AYRES JUNIOR, M.; AYRES, D. L.; SANTOS, A. A. S. Bioestat 5.3 Aplicações estatísticas nas áreas das ciências biológicas e médicas. Belém: IDSM, 2007.

ANDRADE, F. A. G.; GOMES, M. N.; UIEDA, W.; BEGOT, A. L.; RAMOS, O. S.; FERNANDES, M. E. B.. Geographical analysis for detecting high-risk areas for bovine/human rabies transmitted by the common hematophagous bat in the Amazon region, Brazil. PLOS ONE, San Francisco, v.11, n.7, p.1-15, 2016. DOI:

https://doi.org/10.1371/journal.pone.0157332

BLACKWOOD, J. C.; STRICTER, D. G.; ALTIZER, S.; ROHANI, P.. Resolving the roles of immunity, pathogenesis, and immigration for rabies persistence in vampire bats. Proceeding of the National Academy of Sciences of the United States of America, v.110, n.51, p.20837-20837, 2013. DOI: https://doi.org/10.1073/pnas.1308817110

BRAGA, G. B.; GRISI-FILHO, J. H.; LEITE, B. M.; SENA, E. F.; DIAS, R. A.. Predictive qualitative risk model of bovine rabies occurrence in Brazil. Preventive Veterinary Medicine, v.113, n.4, p.536-546, 2014. DOI:

https://doi.org/10.1016/j.prevetmed.2013.12.011

BRASIL. Ministério da Agricultura, Pecuária e Abastecimento. Controle da raiva dos herbívoros: Manual Técnico. Brasília: Secretaria de Defesa Agropecuária, 2009.

CASSEB, L. M. N.; BARBOSA, T. F. S.; PEREIRA, A. S.; VIEIRA, C. A.; MEDEIROS, D. B. A.; VASCONCELOS, P. F. C.; TRAVASSOS DA ROSA, E. S.; CASSEB, A. R.. Prevalência de raiva animal em amostras procedentes da região Norte do Brasil, diagnosticadas no Instituto Evandro Chagas no período de 2000 a 2004. Revista Ciências Agrárias, v.46, p.261-274, 2006.

COSTA, L. J. C.. Composição da quiropterofauna e sua soroprevalência de anticorpos contra o vírus da raiva no Nordeste do Pará. Dissertação (Mestrado em Biologia Ambiental) - Universidade Federal do Pará, Belém, 2011.

CORDEIRO, I. M. C. C.; ARBAGE, M. J. C.; SCHWARTZ, G.. Nordeste do Pará: configuração atual e aspectos identificários. In: CORDEIRO, I. M. C. C.; RANGELVASCONCELOS, L. G. T.; SCHWARTZ, G.; OLIVEIRA, F. A.. Nordeste Paraense: panorama geral e uso sustentável das florestas secundárias. Belém: EDUFRA, 2017. p.19-58.

DIOGO FILHO, A. A.. O uso da terra e a distribuição dos casos de raiva bovina em Goiás, Brasil, 1985 a 2016. Dissertação (Mestrado em Conservação de Recursos Naturais do Cerrado) - Instituto Federal Goiano, Goiânia, 2018.

DOGNANI, R.; PIERRE, E. J.; SILVA, M. C. P.; PATRÍCIO, M. A. C.; COSTA, S. C.; PRADO, J. R.; LISBÔA, J. A. N.. Epidemiologia descritiva da raiva dos herbívoros notificados no estado do Paraná entre 1977 e 2012. Pesquisa Veterinária Brasileira, v.36, n.1, p.1145-1154, 2016. DOI:

\section{https://doi.org/10.1590/s0100-736×2016001200001}

FAPESPA. Fundação Amazônia de Amparo a Estudos e Pesquisas. Estatísticas Municipais Paraenses: Bragança, 2016. Belém, 2016.

FERNANDES, M. E. B.; COSTA, L. J.; ANDRADE, F. A. G.; SILVA, L. P.. Rabies in humans and non-human in the state of Pará. Brazilian Journal Infectious Diseases, Salvador, v.17, n.2, p.251-253, 2013. DOI: https://doi.org/10.1016/i.bjid.2012.10.015

GOMES, M. N.; MONTEIRO, A. M. V.. Raiva bovina no estado de São Paulo e sua distribuição espacial entre 1992 e 2003. Arquivo Brasileiro de Medicina Veterinária e Zootecnia, v.63, n.2, p.279-286, 2011. DOI: https://doi.org/10.1590/S0102-09352011000200002

IBGE. Instituto Brasileiro de Geografia e Estatística. População. Rio de Janeiro: IBGE, 2018a.

IBGE. Instituto Brasileiro de Geografia e Estatística. Pesquisa da Pecuária Municipal: Efetivo dos rebanhos, por tipo de rebanho. Rio de Janeiro: IBGE, 2018b.

INPE. Instituto Nacional de Pesquisas Espaciais. 'PRODES Digital': Desmatamento nos municípios da Amazônia Legal. São José dos Campos: INPE, 2018.

LIMA, G. B.. Distribuição espacial e temporal do vírus rábico em humanos e não-humanos no estado do Pará, no período de 1999 a 2005. Monografia (Bacharelado) Universidade Federal do Pará, Belém, 2006.

MAYEN, F.. Haematophagous bats in Brazil, their roles in rabies transmission, impacto no public health, livestock industry and alternatives to on indiscriminate reduction of bat population. Journal of Veterinary Medicine B Infectious Diseases and Veteterinary Public Health, v.50, n.10, p.469472, 2003. DOI: https://doi.org/10.1046/j.1439$\underline{0450.2003 .00713 . x}$

MORAES, B. C.; COSTA, J. M. N.; COSTA, A. C. L.; COSTA, M. H.. Variação espacial e temporal da precipitação no Estado do Pará. Acta Amazonica, v.35, n.2, p.207-214, 2005. DOI: https://doi.org/10.1590/S0044-59672005000200010

MARCOLONGO-PEREIRA, C.; SALLIS, E. S. V.; GRECCO, F. B.; RAFFI, M. B.; SOARES, M. P.; SCHILD, A. L.. Raiva em bovinos na Região Sul do Rio Grande do Sul: epidemiologia e diagnóstico imuno-histoquímico. Pesquisa Veterinária Brasileira, v.31, n.4, 2011. DOI: http://dx.doi.org/10.1590/S0100-736X2011000400010

NEVES, P. A. P. F. G.; SILVA, L. M.; PONTES, A. N.; PAULA, M. T.. Correlação entre pecuária e desmatamento em municípios da mesorregião sudeste do estado do Pará, Brasil. Ambiência Revista do Setor de Ciências Agrárias e Ambientais, v.10, n.3, p.795-806, 2014. DOI: https://doi.org/10.5935/ambiencia.2014.03.11

PARÁ. Portaria n. 05, de 14 de junho de 2004. Resolve disciplinar o Programa Estadual de Controle da Raiva dos Herbívoros (PECRH). Belém: DOE, 2004. 
PUGA, L. C. H. P.. Modelagem espacial da ocorrência de mordeduras de morcegos hematófagos na Zona da Mata de Minas Gerais. Tese (Doutorado em ciência) - Universidade Federal de Viçosa, Viçosa, 2015.

RIBEIRO, T. M. P.; TEIXEIRA, D. G.; REIS, T. S.; SANTOS, H. D.; MOURA, L. T. S.; BRASILEIRO, A. C. M.. Casos notificados de raiva em bovinos na região norte do Brasil no período de 2005 - 2017. Jornal Interdisciplinar de Biociências, v.33, n.1, p.42-46, 2018.

SILVA, G. C. P.; SANTOS, R. F.; ROCHA, S. M.; REIS, V. G. L.; SANTOS, G. R.; GODOY, H. P.; MATHIAS, L. A.; NOCITI, D. L. $P$.. Perfil da ocorrência de raiva animal em diferentes espécies no estado de Mato Grosso, Brasil, de 2002 a 2011.
Revista Brasileira de Ciência Veterinária, v.24, n.3, p.151156, 2017. DOI: https://doi.org/10.4322/rbcv.2017.029

SOUZA, P.; AMARAL, B.; GITTI, C.. Raiva animal na cidade do Rio de Janeiro: emergência da doença em morcegos e novos desafios para o controle. Revista Instituto Adolfo Lutz, v.73, n.1, p.119-124. 2014. DOI: https://doi.org/10.18241/0073$\underline{98552014731596}$

TEIXEIRA, L. H. M.; TOMAZ, L. A. G.; LINHARES, G. F. C.; SANTOS, M. F. C.; JAYME, V. S.. Distribuição espaço-temporal dos diagnósticos laboratoriais da raiva animal. Ciência Animal Brasileira, Goiânia, v.16, n.1, p.144-157, 2015. DOI: http://dx.doi.org/10.1590/1809-6891v16i131282

A CBPC - Companhia Brasileira de Produção Científica (CNPJ: 11.221.422/0001-03) detém os direitos materiais desta publicação. Os direitos referem-se à publicação do trabalho em qualquer parte do mundo, incluindo os direitos às renovações, expansões e disseminações da contribuição, bem como outros direitos subsidiários. Todos os trabalhos publicados eletronicamente poderão posteriormente ser publicados em coletâneas impressas sob coordenação da Sustenere Publishing, da Companhia Brasileira de Produção Científica e seus parceiros autorizados. Os (as) autores (as) preservam os direitos autorais, mas não têm permissão para a publicação da contribuição em outro meio, impresso ou digital, em português ou em tradução. 